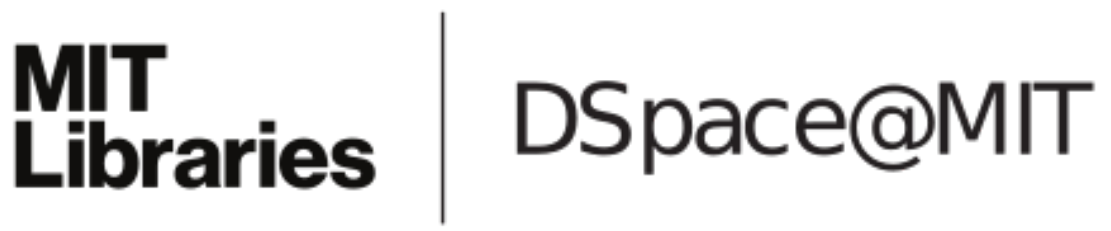

\author{
MIT Open Access Articles
}

Thermoelectric properties of nanoporous Ge

The MIT Faculty has made this article openly available. Please share how this access benefits you. Your story matters.

Citation: Lee, Joo-Hyoung, and Jeffrey C. Grossman. “Thermoelectric properties of nanoporous Ge." Applied Physics Letters 95, no. 1 (2009): 013106. ( 2009 American Institute of Physics

As Published: http://dx.doi.org/10.1063/1.3159813

Publisher: American Institute of Physics (AIP)

Persistent URL: http://hdl.handle.net/1721.1/79650

Version: Final published version: final published article, as it appeared in a journal, conference proceedings, or other formally published context

Terms of Use: Article is made available in accordance with the publisher's policy and may be subject to US copyright law. Please refer to the publisher's site for terms of use. 


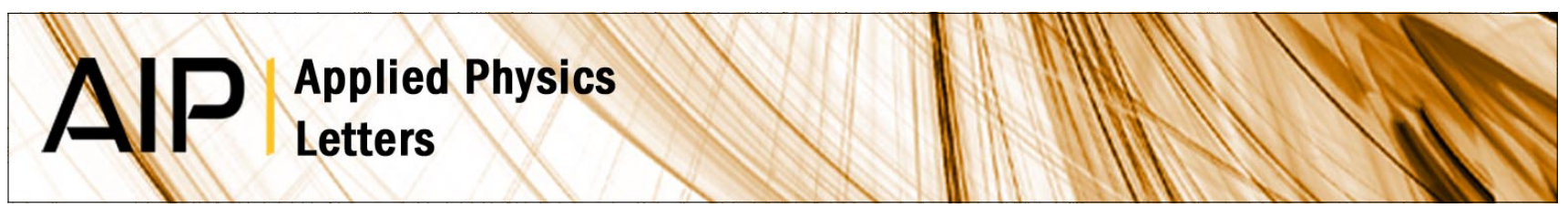

\section{Thermoelectric properties of nanoporous $\mathrm{Ge}$}

Joo-Hyoung Lee and Jeffrey C. Grossman

Citation: Appl. Phys. Lett. 95, 013106 (2009); doi: 10.1063/1.3159813

View online: http://dx.doi.org/10.1063/1.3159813

View Table of Contents: http://apl.aip.org/resource/1/APPLAB/v95/i1

Published by the AIP Publishing LLC.

Additional information on Appl. Phys. Lett.

Journal Homepage: http://apl.aip.org/

Journal Information: http://apl.aip.org/about/about_the_journal

Top downloads: http://apl.aip.org/features/most_downloaded

Information for Authors: http://apl.aip.org/authors

\section{ADVERTISEMENT}

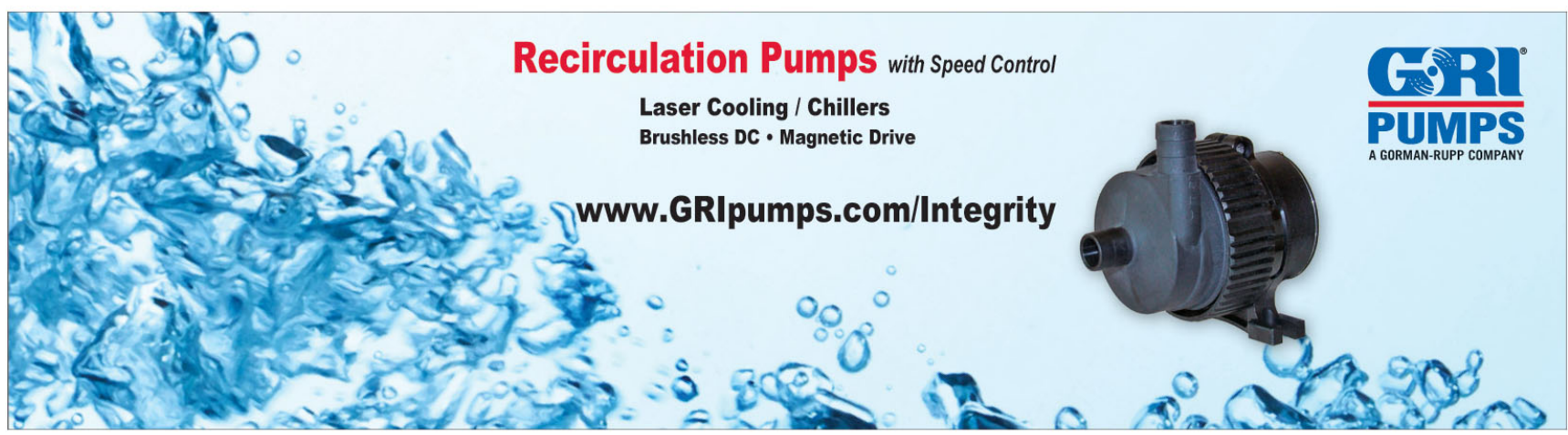




\title{
Thermoelectric properties of nanoporous Ge
}

\author{
Joo-Hyoung Lee ${ }^{1, a)}$ and Jeffrey C. Grossman ${ }^{1,2, b)}$ \\ ${ }^{1}$ Berkeley Nanosciences and Nanoengineering Institute, University of California, Berkeley, \\ California 94720, USA \\ ${ }^{2}$ Department of Materials Science and Engineering, Massachusetts Insitute of Technology, \\ 77 Massachusetts Avenue, Cambridge, Massachusetts 02139-4307, USA
}

(Received 22 April 2009; accepted 5 June 2009; published online 7 July 2009)

\begin{abstract}
We computed thermoelectric properties of nanoporous $\mathrm{Ge}$ (np-Ge) with aligned pores along the [001] direction through a combined classical molecular dynamics and first-principles electronic structure approach. A significant reduction in the lattice thermal conductivity of np-Ge leads to a 30 -fold increase in the thermoelectric figure-of-merit $(Z T)$ compared to that of bulk. Detailed comparisons with the recently proposed np-Si show that although the maximum $Z T\left(Z T_{\max }\right)$ of Ge is nine times larger than that of $\mathrm{Si}$ in the bulk phase, $Z T_{\max }$ of np-Ge is twice as large as that of np-Si due to the similarity in lattice thermal conductivity of the two np systems. Moreover, $Z T_{\max }$ is found to occur at a carrier concentration two orders of magnitude lower than that for with np-Si due to the dissimilarities in their electronic structure. (C) 2009 American Institute of Physics.
\end{abstract}

[DOI: $10.1063 / 1.3159813$ ]

Thermoelectricity, or solid-state energy conversion between temperature gradient and electricity, has received much attention recently due to its promise for environmentally benign power generation and cooling applications. ${ }^{1}$ However, a large enhancement in the energy conversion efficiency is still needed ${ }^{2,3}$ in order to utilize thermoelectric (TE) materials as an abundant resource, and as such, intense research efforts have focused on increasing the TE figure-ofmerit $Z T=S^{2} \sigma T / \kappa$, by which the efficiency of TE materials is described. Here, $S$ is the Seebeck coefficient, $\sigma$ is the electrical conductivity, $\kappa$ is the sum of the electronic and lattice thermal conductivities ( $\kappa_{e}$ and $\kappa_{1}$, respectively), and $T$ is the absolute temperature.

Two approaches are commonly used to increase $Z T$. In one approach, the electronic density of states (DOS) is engineered to have a sharp singularity near the Fermi energy $E_{\mathrm{F}}$, which has been shown to lead to a direct increase in the power factor $S^{2} \sigma{ }^{4}$ This approach can be realized by modifying the DOS through doping, for example, with $\mathrm{Tl}$ in PbTe to achieve $Z T \sim 1.5$ at $775 \mathrm{~K}$, a factor of two improvement over the best $p$-type PbTe-based TE materials $\left(\mathrm{Na}_{0.01} \mathrm{~Pb}_{0.99} \mathrm{Te}\right){ }^{5}$. Another approach for increasing $Z T$ is through reducing $\kappa$, which has greatly benefitted from recent advances in semiconductor nanostructure synthesis. For example, while considered a poor TE material in the bulk phase $(Z T<0.01$ at $300 \mathrm{~K})$, Si was recently shown experimentally to have significantly increased $Z T$ values in the form of nanowires $(Z T \sim 0.6$ at $300 \mathrm{~K}),{ }^{6,7}$ largely due to the sharp decrease in $\kappa$ (from $150 \mathrm{~W} / \mathrm{mK}$ in the bulk to $0.76-1.6 \mathrm{~W} / \mathrm{mK}$ in the nanowires). Wang et al. ${ }^{8}$ also reported that nanopowders of $n$-type $\mathrm{SiGe}$ possess $\kappa$ $\sim 2.5 \mathrm{~W} / \mathrm{mK}$, lower than that of the conventional bulk alloys $(4.6 \mathrm{~W} / \mathrm{mK})$, resulting in a maximum $Z T$ of 1.3 at 1173 K. Recently, Si with vertically aligned nanometer-sized pores (nanoporous $\mathrm{Si}$, or $\mathrm{np}-\mathrm{Si}$ ) was predicted to have substantially enhanced $Z T$ values at room temperature, mainly due to the

\footnotetext{
a) Electronic mail: jhyoung73@ berkeley.edu.

${ }^{b)}$ Electronic mail: jcg@mit.edu.
}

significant reduction in lattice thermal conductivity. ${ }^{9}$ The use of aligned nanopores for reducing $\kappa_{1}$ may have important advantages in manufacturing, doping, and the formation of robust contacts with electrical leads, compared to other nanostructuring approaches due the bulk character of the host material. It is therefore of interest to further explore the potential of such nanoporous (np) materials for TE applications. In particular, given the higher carrier mobility and lower thermal conductivity of bulk Ge compared to $\mathrm{Si}$, as well as the widespread use of SiGe alloys in existing TE applications,${ }^{10-12}$ understanding the properties of np-Ge represents a valuable next step.

In this work, the TE properties of $\mathrm{np}-\mathrm{Ge}$ are computed using a combined density functional theory and classical molecular dynamics (MD) approach. We note that a straightforward extrapolation from bulk behavior to the nanoscale is not necessarily reliable, and indeed our results show that the highest $Z T$ value, $Z T_{\max }$, for np-Ge is twice that of np-Si, which is far less than the ninefold increase in the respective bulk phases. The striking difference in the enhancement of $Z T_{\max }$ between the respective bulk and np phases is due to the similarity of $\kappa_{1}$ in the np geometry; while $\kappa_{1}$ of bulk Ge is three times lower than that of bulk $\mathrm{Si}$, our calculations show that $\mathrm{np}-\mathrm{Ge}$ and $\mathrm{np}-\mathrm{Si}$ have very similar $\kappa_{\mathrm{l}}$. In contrast, the power factor, $S^{2} \sigma$, of bulk Ge is 2.5 times larger than that of bulk $\mathrm{Si}$, and np-Ge shows a similar increase in $S^{2} \sigma$ over $\mathrm{np}-\mathrm{Si}$, which suggests that $Z T_{\max }$ of different np systems is almost entirely determined by the differences in their electronic properties. Further, our calculations show that np-Ge may have advantages over np-Si since the carrier concentration for $Z T_{\max }$ is two orders of magnitude lower, less than $10^{18} \mathrm{~cm}^{-3}$ because of the high dispersiveness in the band structure in np-Ge.

As in the np-Si cases, the structure of np-Ge employed in the present study contains periodically arranged cylindrical pores along the $\mathrm{Ge}[001]$ direction in a square lattice pattern. The pore diameter, $d_{\mathrm{p}}$, and pore spacing, $d_{\mathrm{s}}$, are varied separately in order to examine the dependence of the transport coefficients on these variables. 

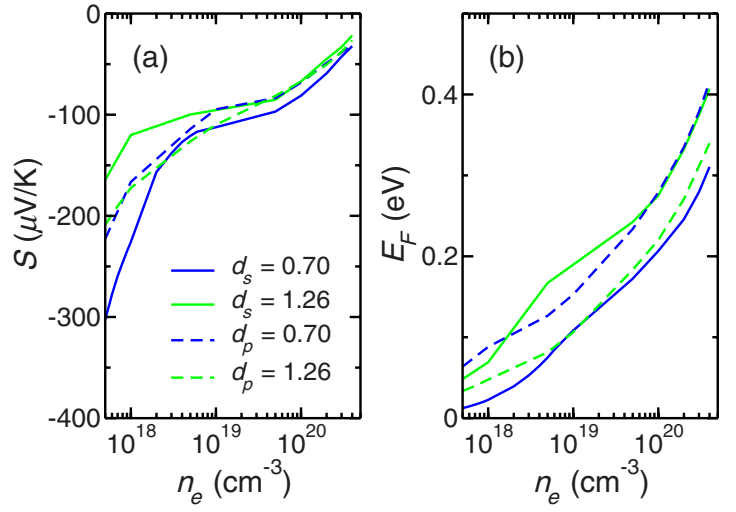

FIG. 1. (Color online) (a) Room-temperature Seebeck coefficients of np-Ge for different $\left(d_{\mathrm{p}}, d_{\mathrm{s}}\right)(\mathrm{nm})$ values and (b) Fermi energy calculated from the CBM. Solid (dotted) lines represent $d_{p}\left(d_{\mathrm{s}}\right)=1.0 \mathrm{~nm}$ cases.

Following the same computational approach as in Ref. 13 , the lattice thermal conductivity perpendicular to the pore axis $\left(\kappa_{1}^{\perp}\right)$ is calculated through a series of classical MD simulations using a Tersoff potential, ${ }^{14,15}$ and is found to be greatly reduced in the presence of pores from the bulk value which is computed to be $90 \pm 6 \mathrm{~W} / \mathrm{mK}$, about $50 \%$ higher than the experimental value. ${ }^{16}$ At $\left(d_{\mathrm{p}}, d_{\mathrm{s}}\right)=(1.0,0.70) \mathrm{nm}$, the smallest $\kappa_{1}^{\perp}=0.51 \pm 0.03 \mathrm{~W} / \mathrm{mK}$ is obtained, which is 180 times smaller than the bulk value. Similar to the np-Si cases, ${ }^{13}$ the reduction in $\kappa_{1}^{\perp}$ for np-Ge originates from the increased phonon-phonon scattering and phonon-pore surface scattering.

The electronic transport coefficients are calculated in terms of the so-called "transport distribution function",

$$
\Xi(\varepsilon)=\tau \sum_{n} \int \frac{d \mathbf{k}}{4 \pi^{3}} v_{n \mathbf{k}} v_{n \mathbf{k}} \delta\left(\varepsilon-\varepsilon_{n \mathbf{k}}\right)
$$

and

$$
\mathbf{L}^{(\alpha)}=e^{2} \int d \varepsilon[-\partial f(\varepsilon) / \partial \varepsilon] \Xi(\varepsilon)(\varepsilon-\mu)^{\alpha} .
$$

Here, $\varepsilon_{n k}$ is a single particle energy eigenvalue, obtained through electronic structure calculations, in the $n$th band at $\mathbf{k}, v_{n \mathbf{k}}=(1 / \hbar) \nabla_{\mathbf{k}} \varepsilon_{n \mathbf{k}}, f(\varepsilon)$ the Fermi function and $\mu$ the chemical potential, respectively. Computational details of the electronic structure calculations are described elsewhere. Note that while considered to be energy independent, the relaxation time $\tau$ is a function of carrier concentration obtained by fitting the experimental mobility values for bulk Ge. ${ }^{17}$

Figure 1(a) shows the calculated Seebeck coefficient for np-Ge as a function of carrier concentration $n_{e}$. As is clear from the figure, the magnitude of the Seebeck coefficient increases as $n_{e}$ decreases, which can be understood by examining the Fermi energy $\left(E_{\mathrm{F}}\right)$, measured from the conduction band minimum (CBM) as a function of $n_{e}$, as shown in Fig. 1(b). ${ }^{18}$ As can be seen, $E_{\mathrm{F}}$ moves closer to the CBM as $n_{e}$ decreases, which leads to an enhanced asymmetry in the single particle energy distribution with respect to $E_{\mathrm{F}}$ within the conduction band, resulting in an increase of $\mathbf{L}^{(1)}$ and thus $|S|$. As the carrier concentration becomes high, however, $E_{\mathrm{F}}$ is located far above the CBM, causing the single particle
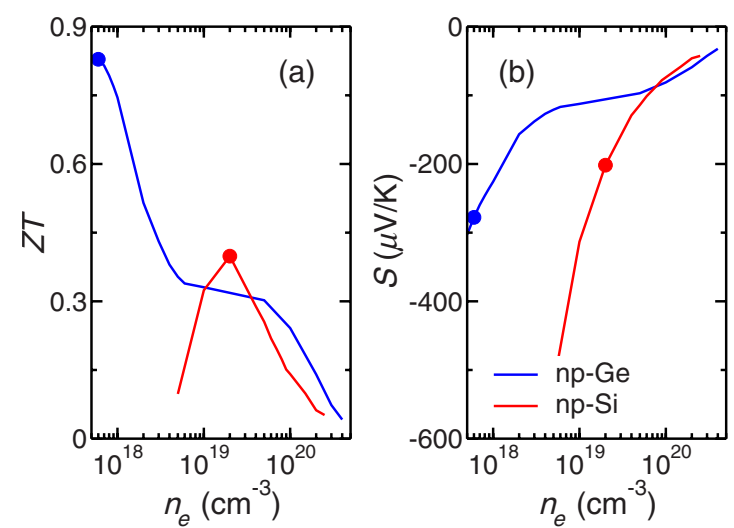

FIG. 2. (Color online) (a) ZT and (b) $S$ for np-Ge (blue) and np-Si (red), respectively. Circles represent $Z T_{\max }$ for the two systems in (a) and the corresponding $S$ values in (b), respectively. Results are presented only for the geometries that produce maximum $Z T$.

energy to be symmetrically distributed around $E_{\mathrm{F}}$, which results in a decrease in $\mathbf{L}^{(1)}$ (and in $|S|$ ), and $|S|$ thus becomes similar even for different $\left(d_{\mathrm{p}}, d_{\mathrm{s}}\right)$ values.

Figure 2(a) shows calculated $Z T_{\max }$ values of np-Ge together with those of $\mathrm{np}-\mathrm{Si}$ as a function of carrier concentration. Here, we present results only for the pore structures that give maximum $\mathrm{ZT}$ for $\mathrm{np}-\mathrm{Ge}$ and $\mathrm{np}-\mathrm{Si}$, respectively, in order to focus on the comparison between the two systems. As can be seen from the figure, $Z T$ of np-Ge decreases as $n_{e}$ increases mainly due to the decrease in $|S|$. We also find that $Z T_{\max }$ of 0.83 occurs at a low carrier concentration, $n_{e}=6.0$ $\times 10^{17} \mathrm{~cm}^{-3}$. This $Z T_{\max }$ is about 30 times larger than the corresponding bulk value, which is computed to be 0.026 in the present study. The large enhancement in $Z T$ is due to the substantial reduction in $\kappa_{1}$ as in the np-Si cases. We note that the computational approach employed in the present work together with the use of the Tersoff potential is likely to underestimate the predicted $Z T_{\max }$ approximately by a factor of two. ${ }^{9}$

Further comparison between the present results and those of np-Si are given in Table I. We note that Ge has a value for $\kappa_{1}$ that is three times lower than $\mathrm{Si}$ in the bulk phase. This small $\kappa_{1}$, together with a 2.5 times larger power factor, leads to a nearly nine times higher $Z T_{\max }$ value for bulk Ge than bulk Si. It would be, therefore, expected that np-Ge also shows lower $\kappa_{1}^{\perp}$ and higher $Z T_{\max }$ values compared to those of np-Si by similar factors as in bulk. As is clear from the table, however, this extrapolation from the bulk behavior to the nanoscale does not hold: while there is a factor of three difference in the bulk phase, $\kappa_{1}^{\perp}$ at $Z T_{\max }$ is similar for both np-Ge $(0.51 \mathrm{~W} / \mathrm{mK})$ and np-Si $(0.62 \mathrm{~W} / \mathrm{mK})$,

TABLE I. Room-temperature $\kappa_{\mathrm{l}}(\mathrm{W} / \mathrm{mK}), \quad S^{2} \sigma \quad\left(10^{-2} \mathrm{~W} / \mathrm{mK}^{2}\right), \quad n_{e}$ $\left(10^{19} \mathrm{~cm}^{-3}\right)$, and $Z T_{\max }$ of $\mathrm{Ge}$ and $\mathrm{Si}$ in bulk and np phases. The minimum $\kappa_{1}^{\perp}$ values are presented for np structures, and values of $S^{2} \sigma$ and $n_{e}$ are given at $Z T_{\max }$. A range of $n_{e}$ is given for bulk Ge and np-Si since $Z T$ is essentially the same for the whole range.

\begin{tabular}{lccccl}
\hline \hline & & $\kappa_{1}\left(\kappa_{1}^{\perp}\right)$ & $S^{2} \sigma$ & $n_{e}$ & $Z T_{\max }$ \\
\hline \multirow{2}{*}{ Bulk } & $\mathrm{Ge}$ & 90 & 0.79 & $0.08-0.1$ & 0.026 \\
& $\mathrm{Si}$ & 273 & 0.31 & 10.0 & 0.003 \\
$\mathrm{np}$ & $\mathrm{Ge}$ & 0.51 & 0.19 & 0.06 & 0.83 \\
& $\mathrm{Si}$ & 0.62 & 0.10 & $2.0-15.0$ & 0.40 \\
\hline \hline
\end{tabular}


(a)

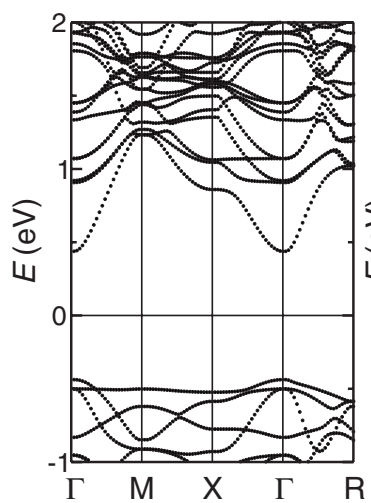

(b)

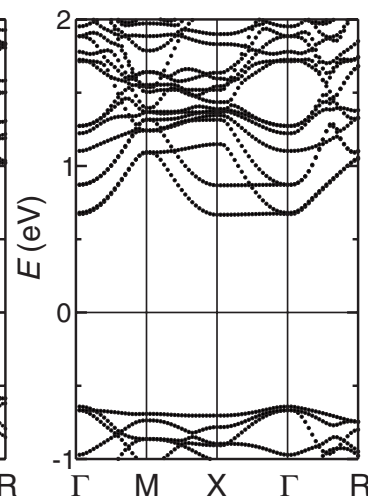

FIG. 3. Single particle band structures for (a) np-Ge and (b) np-Si. The band structures are presented for the $\left(d_{\mathrm{p}}, d_{\mathrm{s}}\right)$ value that produces the highest $Z T$.

which, combined with $S^{2} \sigma$, makes $Z T_{\max }$ of np-Ge twice as large as that of np-Si, as opposed to the factor of 9 in bulk.

The differences in $Z T_{\max }$ between the two np systems result from the dissimilarity in $S$ as presented in Fig. 2(b). In np-Ge, $S$ is $38 \%$ larger in magnitude at $Z T_{\max }$ than that of np-Si, which is due to the different locations of $E_{\mathrm{F}}$ as discussed above, i.e., $E_{\mathrm{F}}$ of np-Si is higher than that of np-Ge by $30 \%$, causing the corresponding $|S|$ to be smaller. Given that the difference in $\sigma$ at $Z T_{\max }$ is less than $2 \%$ between the two systems, the larger $|S|$ of np-Ge leads to an increase in the power factor by $90 \%$ (Table I) and thus in $Z T$ compared to np-Si. We note that although the power factor of np systems becomes reduced from the bulk values, the ratio in $S^{2} \sigma$ between $\mathrm{Ge}$ and $\mathrm{Si}$ is relatively similar for both bulk and np phases, which suggests that electronic properties play a decisive role in determining $Z T_{\max }$ in different np systems.

Another finding of interest is that for $\mathrm{np}-\mathrm{Ge}$, the carrier concentration for $Z T_{\max }\left(6.0 \times 10^{17} \mathrm{~cm}^{-3}\right)$ is much smaller than that of np-Si, for which $Z T$ has a maximum at $n_{e}=2.0$ $\times 10^{19} \mathrm{~cm}^{-3}$. As can be seen from Table I, these values are close to those for bulk in both $\mathrm{Ge}$ and $\mathrm{Si}$ cases. The difference in the carrier concentration between np-Ge and $\mathrm{np}-\mathrm{Si}$ can be explained by considering the electronic band structure, as shown in Fig. 3. As is seen from Fig. 3(a), the lowest conduction band of np-Ge is highly dispersive in all symmetry directions, which is essentially the same as that of the bulk phase, and differences between np and bulk Ge occur only in the upper bands. In contrast, the situation is quite different for the np-Si cases [Fig. 3(b)]: although dispersive as in bulk $\mathrm{Si}$ in the symmetry directions such as $\Gamma \rightarrow M, M$ $\rightarrow X$, and $\Gamma \rightarrow R$, the band in the $\Gamma \rightarrow X$ direction becomes flat in $\mathrm{np}-\mathrm{Si}$, which results in $E_{\mathrm{F}}$ from the CBM to be located lower for $\mathrm{np}-\mathrm{Si}$ than for $\mathrm{np}-\mathrm{Ge}$ at a given carrier concentration. Since np-Si has higher $E_{\mathrm{F}}$ at its $Z T_{\max }$ by $30 \%$ than np-Ge as discussed above, the corresponding carrier concentration is lower for np-Ge than that of np-Si.
Since the present results together with those of np-Si predict that forming regular arrays of nanopores might be an effective approach for increasing $Z T$ significantly, one might be tempted to carry out such a process for a well-known TE material such as $\mathrm{Bi}_{2} \mathrm{Te}_{3}$ in order to improve its TE efficiency. However, this may not be the case: since bulk $\mathrm{Bi}_{2} \mathrm{Te}_{3}$ already has a low $\kappa_{\mathrm{l}} \sim 1.5 \mathrm{~W} / \mathrm{mK},{ }^{19}$ the reduction in $\kappa_{\mathrm{l}}$ caused by pores would not be as large as np-Ge or np-Si, and might be only a factor of three or so, based on our findings. In addition, the lowest conduction band of bulk $\mathrm{Bi}_{2} \mathrm{Te}_{3}$ has both highly dispersive and relatively flat parts as in bulk $\mathrm{Si}^{20}$ In the np phase, therefore, $S^{2} \sigma$ is expected to be decreased from the bulk value by a similar factor as in the $\mathrm{Si}$ case, which, together with the decrease in $\kappa_{1}$, would lead to a similar ZT as in bulk or only a marginal increase. Our findings suggest that generating nanopores in semiconductors possessing high power factors with a dispersive lowest conduction band in the bulk phase would be more beneficial in achieving high $Z T$ values.

This work was performed under the auspices of the National Science Foundation (NSF) by the University of California, Berkeley under Grant No. 0425914, and computations were performed at the National Energy Research Scientific Computing Center. Part of this work was supported by NSF through the Network for Computational Nanotechnology, Grant EEC-0634750.

${ }^{1}$ T. M. Tritt and M. A. Subramanian, MRS Bull. 31, 188 (2006).

${ }^{2}$ A. Majumdar, Science 303, 777 (2004).

${ }^{3}$ C. B. Vining, Nature Mater. 8, 83 (2009).

${ }^{4}$ G. D. Mahan and J. O. Sofo, Proc. Natl. Acad. Sci. U.S.A. 93, 7436 (1996).

${ }^{5}$ J. P. Heremans, V. Jovovic, E. S. Toberer, A. Saramat, K. Kurosaki, A. Charoenphakdee, S. Yamanaka, and G. J. Snyder, Science 321, 554 (2008).

${ }^{6}$ A. I. Hochbaum, R. Chen, R. D. Delgado, W. Liang, E. C. Garnett, M. Najarian, A. Majumdar, and P. Yang, Nature (London) 451, 163 (2008).

${ }^{7}$ A. I. Boukai, Y. Bunimovich, J. Tahir-Kehli, J.-K. Yu, W. A. Goddard III, and J. R. Heath, Nature (London) 451, 168 (2008).

${ }^{8}$ X. W. Wang, H. Lee, Y. C. Lan, G. H. Zhu, G. Joshi, D. Z. Wang, J. Yang, A. J. Muto, M. Y. Tang, J. Klatsky, S. Song, M. S. Dresselhaus, G. Chen, and Z. F. Ren, Appl. Phys. Lett. 93, 193121 (2008).

${ }^{9}$ J.-H. Lee, G. A. Galli, and J. C. Grossman, Nano Lett. 8, 3750 (2008).

${ }^{10}$ J.-S. Lin and Y. Miyamoto, J. Mater. Res. 15, 647 (2000).

${ }^{11}$ M. S. Dresselhaus, G. Chen, M. Y. Tang, R. Yang, H. Lee, D. Wang, Z. Ren, J. Fleurial, and P. Gogna, Adv. Mater. (Weinheim, Ger.) 19, 1043 (2007)

${ }^{12}$ N. Mingo, D. Hauser, N. P. Kobayashi, M. Plissonnier, and A. Shakouri, Nano Lett. 9, 711 (2009).

${ }^{13}$ J.-H. Lee, J. C. Grossman, J. Reed, and G. A. Galli, Appl. Phys. Lett. 91, 223110 (2007).

${ }^{14}$ J. Tersoff, Phys. Rev. B 39, 5566 (1989).

${ }^{15}$ C. V. Ciobanu and R. M. Briggs, Appl. Phys. Lett. 88, 133125 (2006).

${ }^{16}$ C. J. Glassbrenner and G. A. Slack, Phys. Rev. 134, A1058 (1964).

${ }^{17}$ D. L. Rode, Phys. Status Solidi B 53, 245 (1972).

${ }^{18}$ D. Vashaee and A. Shakouri, Phys. Rev. Lett. 92, 106103 (2004).

${ }^{19}$ C. B. Satterthwaite and R. W. Ure, Phys. Rev. 108, 1164 (1957).

${ }^{20}$ M. Kim, A. J. Freeman, and C. B. Geller, Phys. Rev. B 72, 035205 (2005). 